\title{
Simulations on Single Vacancy Defect Transients for Face-Center-Cubic Structure
}

\author{
Kwuanchanok Chansawang and Sunchai Nilsuwankosit \\ Department of Nuclear Technology, Faculty of Engineering, \\ Chulalongkorn University, Bangkok 10330, Thailand
}

Received 2012-10-25, Revised 2013-02-15; Accepted 2013-07-20

\begin{abstract}
The simulations on single vacancy defect transients for FCC structure were conducted to study the change in its final structure, especially the average atomic volume. The numerical code "ALINE" was employed for this purpose. The results obtained showed that when a single vacancy defect occurred in a perfect FCCcrystal structure, the average atomic volume was found to be suddenly increased and then gradually decreased down the value close to the initial value. This suggested that the FCC structure was able to expand and fill the volume originally occupied by the missing atom.
\end{abstract}

Keywords: Vacancy Defect, FCC Structure, Transients

\section{INTRODUCTION}

The solid materials are generally observed to have the crystalline structure. The structure is made up from the periodically repeating structural building blocks, the unit cells. The formation of the crystal is typically controlled by the valence electrons of the atoms that form the crystal. The strength, color and other physical properties are then affected by the crystal structure. In general, the crystal contains defects, or imperfections, which locally disrupt the ideal structure of the crystal. These defects can be point defects, line defects or dislocations and surface defects. The defects in the crystal structure can have a tremendous effect on the property of a material. Therefore, the improvements on the physical, electrical, magnetic and optical properties of the crystal materials can be done by controlling the imperfections in their structures. Consequently, many fields of science and engineering are interested in how the defects are formed, how they interact with each other and how they affect macroscopic properties (Kittel, 2004; Ibach and Luth, 2009; Henderson, 1972).
This study was conducted in order to understand the dynamics of the single vacancy defects, specifically its transient. For this purpose, ALINE: Atomic Laboratory for Interactive Numerical Experiments, (Patriarca et al., 2007) was chosen to simulate the transients.

\section{MATERIALS AND METHODS}

\subsection{Simulation of Single Vacancy Defect with ALINE}

ALINE is a three-dimensional interactive molecular dynamics program designed to simulate dynamics of the defects in the crystalline structure. For this study, four scenarios were simulated with ALINE. In general, the material of the study was presumed to have FCC type structure. The kind of atoms or ions that formed the basis of the structure was not specified. While this could have large effect on the length and time scale of the transient, the pattern in which the transient proceeded which was of major interest should be less affected.

For the simulations, the lattice constant was presumed to have the value of unity and the periodic

\section{Corresponding Author: Kwuanchanok Chansawang, Department of Nuclear Technology, Faculty of Engineering,} Chulalongkorn University, Bangkok 10330, Thailand 
condition was used for the boundary condition. The volumes of these systems were of cubical shape initially containing (a) 2000 atoms, (b) 6750 atoms, (c) 16000 atoms and (d) 31250 atoms. In each case, only one atom at the center of the system was assumed to be missing when the simulation was initiated. With the periodic boundary, the different number of atoms contained in the system then in turn identified the defect density in the actual material.

\subsection{Box-Counting Model}

In obtaining the average atomic volume, the volume averagely occupied by one atom, it was necessary to count the number of atoms contained in a given small volume, subsystem and then divide it by the volume. It should be noted that if the subsystem was too small, the number of atoms contained could be varied very widely. On the other hand, if the subsystem was too large then the significance of difference in number of atoms might be lost. It was therefore necessary to choose the subsystem that suited the system. The idea of dividing the system in subsystems or blocks of linear dimension has been applied to a number of problems related to phase transitions since 1964 (Kampen, 1964; Kaski et al., 1984; Rovere et al., 1988).

Since ALINE produced the output which described the positions of atoms at the different time step, by specifying the subsystem as described in Fig. 1, it was then possible to count the number of atoms contained in each subsystem.

\subsection{Average Atomic Volume}

From the distribution of atoms in the system and box-counting model, the information regarding the number of subsystems containing the same number of atoms was obtained and the frequency distribution was created. With i being the specific case where the number of atom $n_{i}$ in the subsystem was counted and $f_{i}$ being the number of such subsystems, the average atomic volume $\mathrm{V}_{\mathrm{m}}$ was calculated by Equation 1:

$\mathrm{V}_{\mathrm{m}}=\frac{\sum_{\mathrm{i}} \mathrm{f}_{\mathrm{i}} \frac{\mathrm{B}_{\mathrm{x}} \mathrm{B}_{\mathrm{y}} \mathrm{B}_{\mathrm{z}}}{\mathrm{n}_{\mathrm{i}}}}{\sum_{\mathrm{i}} \mathrm{f}_{\mathrm{i}}}$

It should be noted that $\sum_{\mathrm{i}} \mathrm{f}_{\mathrm{i}}$ was the total number of subsystems and $\sum_{\mathrm{i}} \mathrm{f}_{\mathrm{i}} \mathrm{n}_{\mathrm{i}}$ was the total number of atoms contained in the whole system.

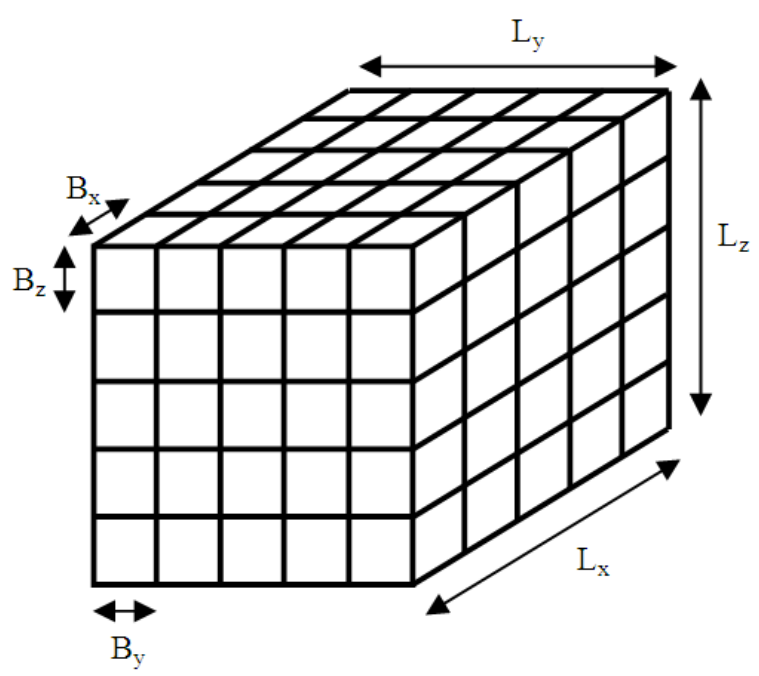

Fig. 1. System of length $L_{x}$, width $L_{y}$ and height $L_{z}$ was divided in to subsystems, each with the length, the width and the height of $B_{x}, B_{y}$ and $B_{z}$ $\left(\mathrm{B}_{\mathrm{x}}=\frac{\mathrm{L}_{\mathrm{x}}}{\mathrm{n}_{\mathrm{d}}}, \mathrm{B}_{\mathrm{y}}=\frac{\mathrm{L}_{\mathrm{y}}}{\mathrm{n}_{\mathrm{d}}}, \mathrm{B}_{\mathrm{z}}=\frac{\mathrm{L}_{\mathrm{z}}}{\mathrm{n}_{\mathrm{d}}}\right)$

\section{RESULTS}

\subsection{Variation of Average Atomic Volume with Number of Subsystems}

From the results regarding the positions of the atoms in Cartesian coordinate, obtained from ALINE, the boxcounting model was used and the average atomic volume was calculated. As it was necessary to identify the suitable size of subsystem which would have allowed for the calculation of the average atomic volume that was both stable (not widely varied by the number of subsystems) but still sensitive to the change in number of atoms that were moving in and out of the subsystems, the counting obtained with different numbers of subsystems $\left(\mathrm{n}_{\mathrm{d}}^{3}\right)$ were obtained and the related average atomic volume were calculated as shown in Fig. 2-5.

From Fig. 2-5, the very large value of $n_{d}$ in every case resulted in the very small average atomic volume. This was because the actual volumes of the subsystems in these cases were so small such that they would either contain only one atom or not at all. This condition highly distorted and under estimated the average atomic volume. On the other hand, with the very low number of $\mathrm{n}_{\mathrm{d}}$, the subsystems became large such that the change in number of atoms it contained became insignificant. It was of interest that a peak in the average atomic volume 
curved was observed in every case. It was tempting to assume that the value of $n_{d}$ associated with such peak gave the suitable of subsystems to be used. However, it must be reminded that with the FCC type of crystalline structure, the cube shape volume of the system meant that the total initial atoms of (a) 2000, (b) 6750, (c) 16000 and (d) 31250 were respectively associated with the arrangement of 2 structure sets; (a) $2(10 \times 10 \times 10)$, (b) $2(15 \times 15 \times 15), \quad$ (c) $2(20 \times 20 \times 20)$ and (d) $2(25 \times 25 \times 25)$. As a result, in each case, the value of $n_{d}$ that was a common denominator to such arrangement should be avoided since it gave the subsystems that coincided with the crystalline arrangements and therefore, might lead to the bias results. This assessment was confirmed as shown by graphs for the systems with no defects in the same figures.

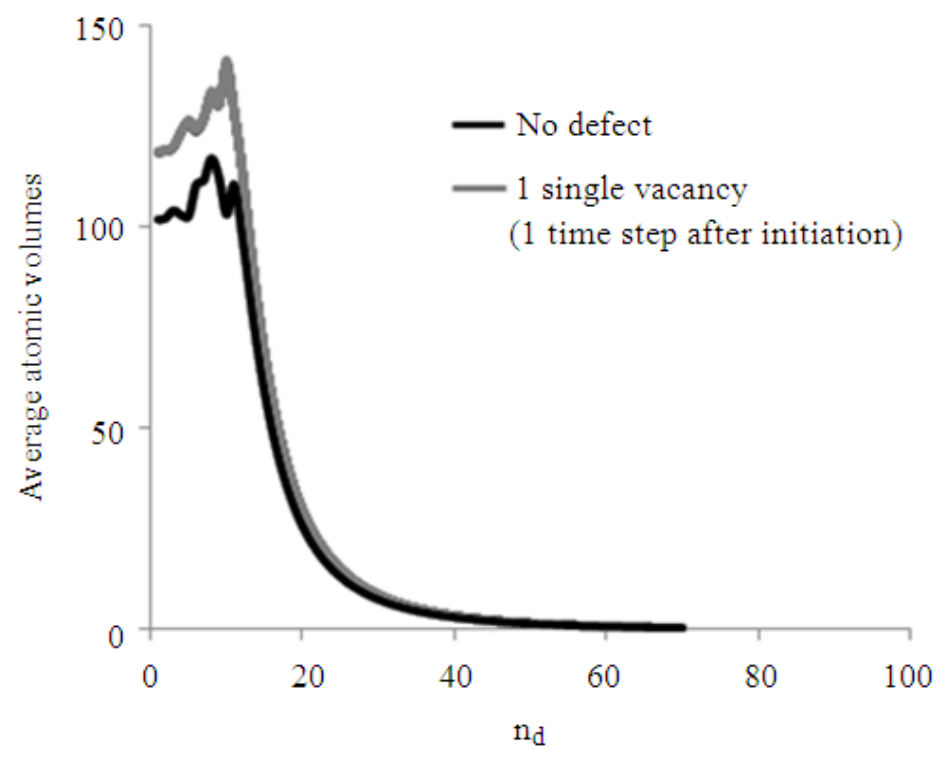

Fig. 2. Variation of average atomic volumes with number of subsystems $\left(\mathrm{n}_{\mathrm{d}}^{3}\right)$; system with initial 2000 atoms

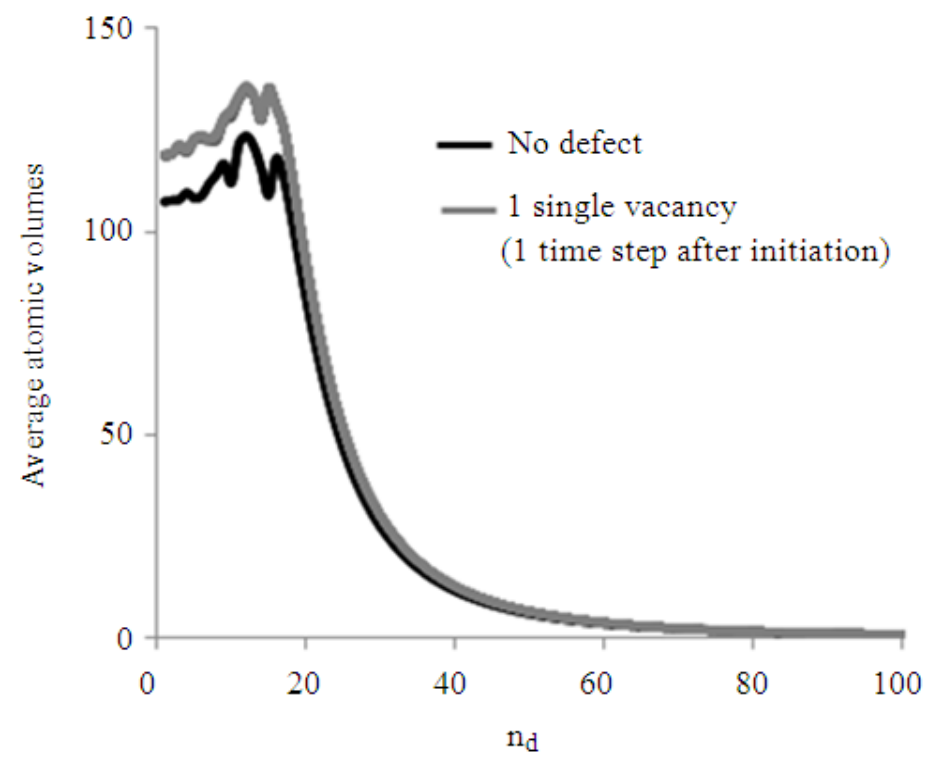

Fig. 3. Variation of average atomic volumes with number of subsystems $\left(\mathrm{n}_{\mathrm{d}}^{3}\right)$; system with initial 6750 atoms 


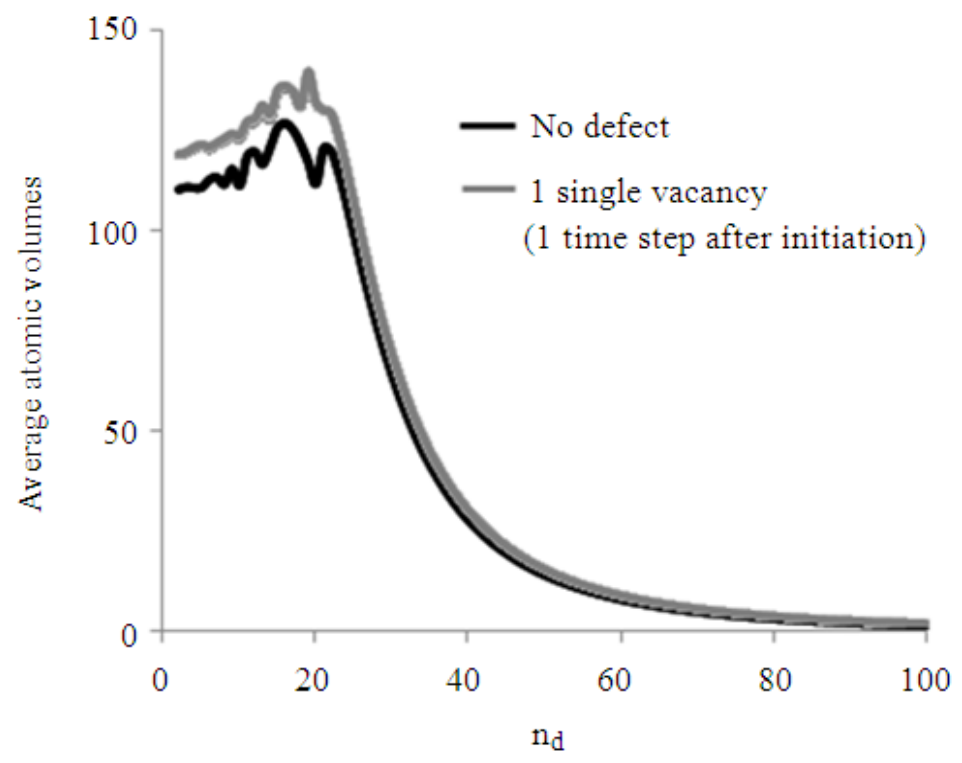

Fig. 4. Variation of average atomic volumes with number of subsystems $\left(\mathrm{n}_{\mathrm{d}}^{3}\right)$; system with initial 16000 atoms

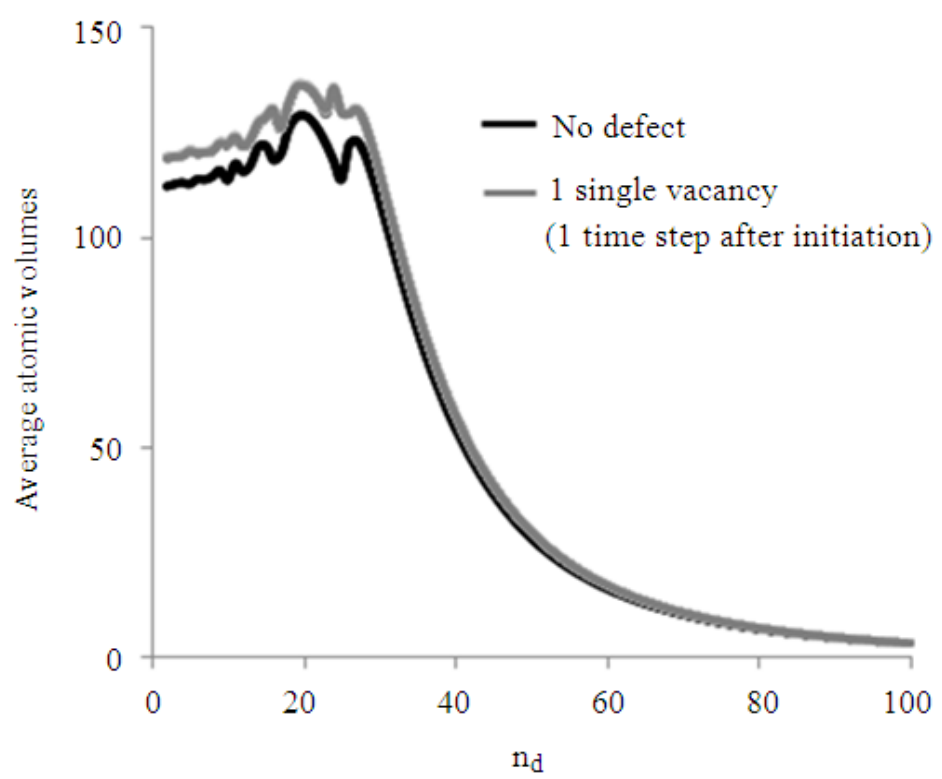

Fig. 5. Variation of average atomic volumes with number of subsystems $\left(n_{d}^{3}\right)$; system with initial 31250 atoms

For the systems with one single vacancy defect, the different value were chosen for $n_{d}$ in each case. For system with the total atoms of $2000, n_{d}$ was 3 . The system with the total atoms of $6750, n_{d}$ was 7 . For the system with the total atoms of $16000, n_{d}$ was 11 and the value of 15 was chosen for $n_{d}$ for the system with the total atoms of 31250 .

\subsection{Transients of Single Vacancy Defects}

With the values for $n_{d}$ as previously described, their transients were simulated. Due to the very large number of atoms involved in the simulation and their complex movements, the change in the average atomic volume was chosen to represent the transient. The results as obtained were shown in Fig. 6. 


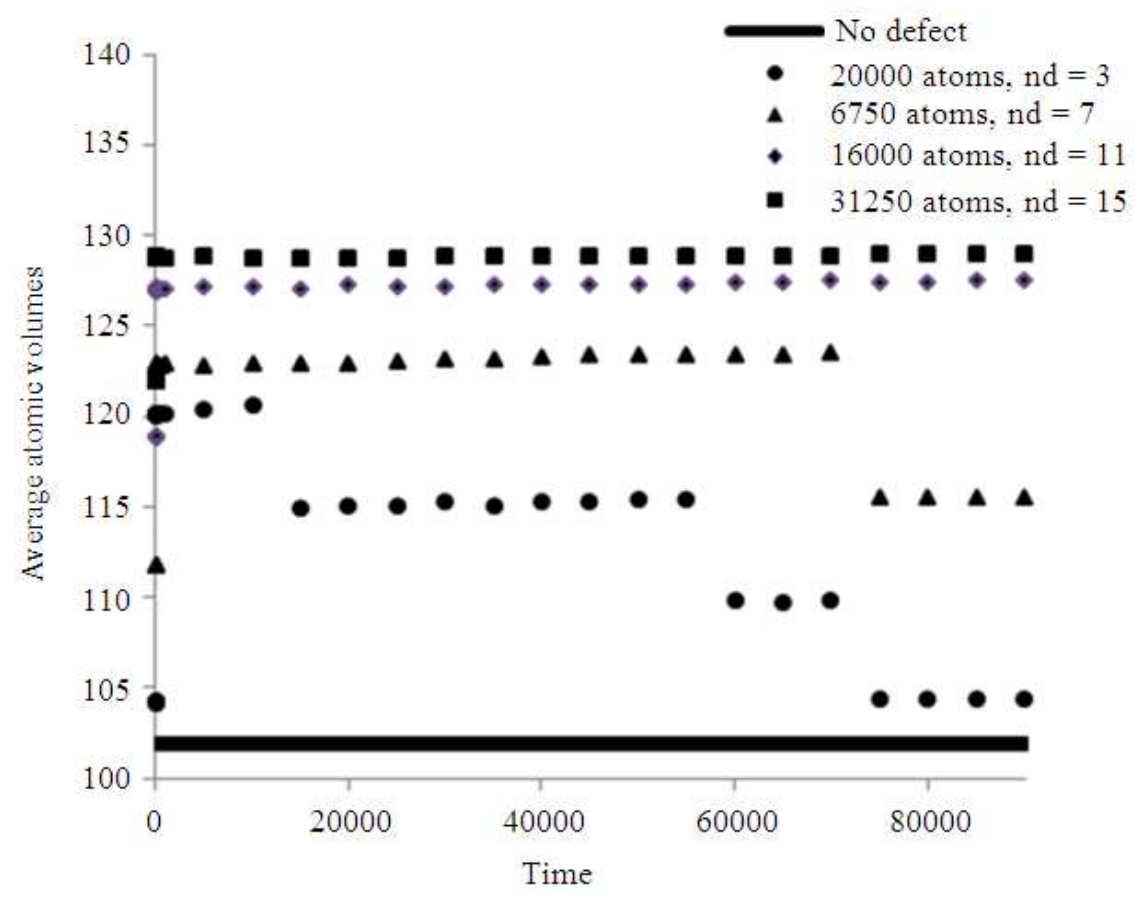

Fig. 6. Change in average atomic volume over times due to single vacancy defect.

It was clearly seen for the cases of 2000 and 6750 initial atoms that the average atomic volumes were suddenly increased. This was due to the space left by the missing atom which allowed the neighbor atoms to expand. The average atomic volume then gradually decreased as the neighboring atoms were expanding to fill such space. It was observed that the process was slower as the total initial atom increased. Considering that the expanding was likely caused by the repulsion between atoms, the longer transient period would suggest that there are the shielding effect that retards the expanding process.

\section{DISCUSSION}

The study on the transient of the single vacancy defect in the crystalline medium of FCC type were conducted. The simulations were performed using the program computer ALINE. Due to the large number of atoms and their complex movements, the change in the average atomic volume over time was used to indicate the process of transient.

It was observed in general that the crystalline medium would expand and eventually fill in the space left by the defect. The actual time needed for the process and the actual size of the defect were not indicated and analyzed. Even though the similar pattern of transients were to be expected, the effect of defect size and the time needed for transient process were still very crucial. The effect of the defect density and/or the size of the system to be simulated were also of concern and required further investigation.

Even more important was how the type of atoms/ions that formed basis of the crystalline structure affect the transient process. Further study in this regard was necessary. In this case, the actual model for the interaction between atoms/ions must be clearly described and verified.

\section{CONCLUSION}

A basic mathematic technique with a numerical method was proposed for studying the point defect in FCC structure. This same technique should be applicable for studying the point defect transient in other structure type. Analysis on the result should help improve the quality of a crystalline materials which relies on the defects in their structure.

\section{ACKNOWLEDGEMENTS}

The researchers are very grateful to the referees for their valuable comments and suggestions to improve the study. 


\section{REFERENCES}

Henderson, B., 1972. Defects in Crystalline Solids. 1st Edn., Edward Arnold, London, ISBN-10: 0713123516, pp: 203.

Ibach, H. and H. Luth, 2009. Solid-State Physics: An Introduction to Principles of Materials Science. 4th Edn., Springer, Berlin, New York, ISBN-10: 3540938044, pp: 533.

Kampen, N.G.V., 1964. Condensation of a classical gas with long-range attraction. Phys. Rev., 135: A362A369. DOI: 10.1103/PhysRev.135.A362

Kaski, K., K. Binder and J.D. Gunton, 1984. Study of cell distribution functions of the three-dimensional Ising model. Phys. Rev. B, 29: 3996-4009.
Kittel, C., 2004. Introduction to Solid State Physics. 8th Edn., John Wiley and Sons, New York, ISBN-10: 047141526X, pp: 704.

Patriarca, M., A. Kuronen, M. Robles and K. Kaski, 2007. Three-dimensional interactive molecular dynamics program for the study of defect dynamics in crystals. Comput. Phys. Commun., 176: 38-47. DOI: 10.1016/j.cpc.2006.07.019

Rovere, M., D.W. Hermann and K. Binder, 1988. Block density distribution function analysis of twodimensional Lennard-Jones fluids. Europhys. Lett., 6: 585-590. 